PROCEEDINGS OF THE

AMERICAN MATHEMATICAL SOCIETY

Volume 125, Number 3, March 1997, Pages 739-745

S 0002-9939(97)03921-X

\title{
GAUSSIAN POLYNOMIALS AND CONTENT IDEALS
}

\author{
WILLIAM HEINZER AND CRAIG HUNEKE
}

(Communicated by Wolmer V. Vasconcelos)

\begin{abstract}
We prove that every regular Gaussian polynomial over a locally Noetherian ring has invertible content ideal. We do this by first proving that Gaussian polynomials over an approximately Gorenstein local ring have principal content ideal. We also show over locally Noetherian rings that a regular polynomial $f$ of degree $n$ is Gaussian if $c(f g)=c(f) c(g)$ for polynomials $g$ of degree at most $n$.
\end{abstract}

\section{INTRODUCTION}

A question ${ }^{1}$ which appears to have originated in the 1965 thesis of Kaplansky's student Tsang asks whether over an integral domain the content of a nonzero Gaussian polynomial is an invertible ideal. Let $R$ be a commutative ring. The content $c(f)$ of a polynomial $f \in R[t]$ is the ideal of $R$ generated by the coefficients of $f$. A polynomial $f(t) \in R[t]$ is Gaussian over $R$ if for every polynomial $g(t) \in R[t]$ we have $c(f g)=c(f) c(g)$.

A recent paper of Glaz and Vasconcelos [GV] on Gaussian polynomials and content ideals has motivated our interest in this topic. Glaz and Vasconcelos prove that a nonzero Gaussian polynomial over a normal Noetherian domain has invertible content ideal [GV, Theorem 4.4]. They also prove that a Gaussian polynomial over an integrally closed quasilocal domain $(R, \mathbf{m})$ has principal content ideal provided the residue field $R / \mathbf{m}$ has characteristic $p>0$ [GV, Theorem 3.1]. We are able to prove that rings that are locally approximately Gorenstein also have this property: a regular Gaussian polynomial over such a ring has invertible content ideal. See Corollary 2.7. Using this we prove that Gaussian polynomials over a locally Noetherian integral domain satisfy the conjecture of Tsang-Glaz-Vasconcelos (cf. Corollary 3.4).

As an extension of the question from Tsang's thesis, it is asked in [AK, Question 1.4] whether over an integral domain $R$ a polynomial $f(t) \in R[t]$ of degree $n$ has an invertible content ideal if $c(f g)=c(f) c(g)$ for all polynomials $g(t) \in R[t]$ of degree at most $n$. We show in Corollary 3.4 that all Noetherian integral domains have this property. Let us say a polynomial $f \in R[t]$ is Gaussian for polynomials of degree at most $m$ if $c(f g)=c(f) c(g)$ for all $g \in R[t]$ with $\operatorname{deg}(g) \leq m$. If $R$ is locally Noetherian and $f(t) \in R[t]$ is of degree $n$ and is regular, we show in

Received by the editors October 18, 1995.

1991 Mathematics Subject Classification. Primary 13A15, 13B25, 13G05, $13 \mathrm{H} 10$.

${ }^{1}$ This question is explicitly given as Conjecture (1.1) in the paper of Sarah Glaz and Wolmer Vasconcelos [GV].

(C)1997 American Mathematical Society 
Theorem 3.3 that if $f$ is Gaussian for polynomials of degree at most $n$, then $f$ is Gaussian and has invertible content ideal.

All rings we consider are assumed to be commutative with identity and our notation is as in $[\mathrm{M}]$.

\section{Gaussian polynomials and approximately Gorenstein Rings}

We begin with several remarks about Gaussian polynomials.

Remark 1.1. Since a finitely generated regular ideal ${ }^{2}$ of a ring $R$ is invertible if and only if it is locally principal, and since a polynomial $f \in R[t]$ is Gaussian if and only if for each maximal ideal $\mathbf{m}$ of $R$, the image of $f$ in $R_{\mathbf{m}}[t]$ is Gaussian over $R_{\mathbf{m}}$, the Tsang-Glaz-Vasconcelos conjecture is equivalent to showing a Gaussian polynomial over a quasilocal integral domain has principal content ideal. Similarly, a polynomial $f \in R[t]$ is Gaussian for polynomials of degree at most $n$ if and only if for each maximal ideal $\mathbf{m}$ of $R$, the image of $f$ in $R_{\mathbf{m}}[t]$ is Gaussian for polynomials of degree at most $n$. It is true over an arbitrary commutative ring $R$ that a polynomial $f(t) \in R[t]$ is Gaussian provided $c(f) R_{\mathbf{m}}$ is a principal ideal of $R_{\mathbf{m}}$ for each maximal ideal $\mathbf{m}$ of $R$. This follows by factoring out the principal content ideal of the image of $f$ in $R_{\mathbf{m}}[t]$ and using in $R_{\mathbf{m}}[t]$ the lemma of Gauss that says that a polynomial with unit content is Gaussian.

Remark 1.2. A useful observation about Gaussian polynomials is that if $f(t) \in R[t]$ is Gaussian over $R$, then for every ideal $I$ of $R$, the image of $f$ in $(R / I)[t]$ is Gaussian over $R / I$. Thus to show a polynomial $f \in R[t]$ is not Gaussian, it suffices to show the existence of an ideal $I$ of $R$ such that the image of $f$ in $(R / I)[t]$ is not Gaussian.

We have found it to be particularly helpful to consider the Gaussian property of polynomials over a zero-dimensional local Gorenstein ring. What makes this case useful for us is (1.2) in conjunction with the fact that an excellent local domain is approximately Gorenstein, where a local Noetherian ring $(R, \mathbf{m})$ is said to be approximately Gorenstein $[\mathrm{H},(1.1)]$ if it satisfies either of the equivalent conditions:

(i) For every integer $N>0$ there is an ideal $I \subseteq \mathbf{m}^{N}$ such that $R / I$ is Gorenstein.

(ii) For every integer $N>0$ there is an $\mathbf{m}$-primary irreducible ideal $I \subseteq \mathbf{m}^{N}$.

Remark 1.3. Hochster proves a local Noetherian $\operatorname{ring}(R, \mathbf{m})$ is approximately Gorenstein if and only if its $\mathbf{m}$-adic completion is approximately Gorenstein $[\mathrm{H}$, (1.2)]. He also proves [H, (1.6)] that an excellent local ring $R$ (so, for example, a complete local Noetherian ring) with $\operatorname{dim}(R) \geq 1$ is approximately Gorenstein if and only if the following two conditions hold:

1. $\operatorname{depth}(R) \geq 1$.

2. If $P \in \operatorname{Ass}(R)$ and $\operatorname{dim}(R / P)=1$, then $R / P \oplus R / P$ is not embeddable in $R$.

Remark 1.4. From (1.3) it follows that an analytically unramified local Noetherian ring $^{3}$ is approximately Gorenstein.

Theorem 1.5. Assume that $R$ is locally ${ }^{4}$ Noetherian and locally approximately Gorenstein. Then every Gaussian polynomial over $R$ has locally principal content ideal.

\footnotetext{
${ }^{2}$ We say an ideal is regular if it contains a regular element, i.e., a non-zero-divisor.

${ }^{3} \mathrm{~A}$ local Noetherian ring $(R, \mathbf{m})$ is said to be analytically unramified if the $\mathbf{m}$-adic completion of $R$ is reduced.

${ }^{4}$ We say a ring $R$ locally has a property if each localization of $R$ at a maximal ideal has the property.
} 
Proof. By using (1.1), we may reduce to the local case and assume that $(R, \mathbf{m})$ is a local Noetherian approximately Gorenstein ring. Suppose $f(t) \in R[t]$ is a Gaussian polynomial. Since $R$ is approximately Gorenstein there exists an $\mathbf{m}$ primary irreducible ideal $I$ of $R$ such that the dimension of $c(f) / \mathbf{m} c(f)$ over the residue field $R / \mathbf{m}$ of $R$ is equal to the dimension of $c\left(f^{*}\right) / \mathbf{m} c\left(f^{*}\right)$ over the residue field of $R / I$, where $f^{*}$ denotes the image of $f$ in $(R / I)[t]$. Therefore $c(f)$ is a principal ideal of $R$ if and only if $c\left(f^{*}\right)$ is a principal ideal of $R / I$. By (1.2), $f^{*}$ is Gaussian over $R / I$, so to prove (1.5) it suffices to prove that a Gaussian polynomial $f$ over a zero-dimensional local Gorenstein ring $R$ has principal content ideal. Let $S=R[t]$. We claim that $\operatorname{Ann}_{S}(f S)=\operatorname{Ann}_{S}(c(f) S)$. As $f \in c(f) S$, the containment $\operatorname{Ann}_{S}(c(f) S) \subseteq \operatorname{Ann}_{S}(f S)$ is clear. Conversely, suppose that $g \in R[t]$ satisfies $f g=0$. Then $c(f g)=0=c(f) c(g)$ and so $c(g) \subseteq \operatorname{Ann}_{R}(c(f))$. As $g \in c(g) S$ it follows that $g \in \operatorname{Ann}_{S}(c(f) S)$. Passing to the zero-dimensional Gorenstein $\operatorname{ring} T=S_{\mathbf{m} S}=R(t)$ and using local duality [BH, Proposition 3.2.12] it then follows that $f T=\operatorname{Ann}_{T}\left(\operatorname{Ann}_{T}(f T)\right)=\operatorname{Ann}_{T}\left(\operatorname{Ann}_{T}(c(f) T)\right)=c(f) T$ so that $c(f) T$ is principal in $T=R(t)$. Since $c(f) T$ is extended from $R$ and $T$ is local, we obtain that $c(f)$ is principal in $R$.

Remark 1.6. The zero-dimensional Noetherian rings over which the content ideal of every Gaussian polynomial is locally principal (or, equivalently, principal) are precisely the ones which are Gorenstein. In view of (1.5), to show this, it suffices to show that a zero-dimensional local ring $(R, \mathbf{m})$ that is not Gorenstein admits a Gaussian polynomial $f \in R[t]$ such that $c(f)$ is not principal. Since 0 is not irreducible in $R$, there exist nonzero elements $x, y \in R$ such that $\mathbf{m} x=\mathbf{m} y=0$, but $x R \neq y R$. Let $f=x t+y \in R[t]$. Then $c(f)$ is not principal, but we claim that $f$ is Gaussian over $R$. For if $g \in R[t]$ and the content of $g$ is not contained in $\mathbf{m}$, then $g$ is Gaussian and $c(f g)=c(f) c(g)$. On the other hand, if $c(g) \subseteq \mathbf{m}$, then $c(g f)=c(f) c(g)=0$. Hence $f$ is Gaussian.

Remark 1.7. The property of a polynomial being Gaussian is not, in general, preserved under polynomial ring extension. If $R$ is a zero-dimensional Noetherian ring which is not Gorenstein, then as noted in (1.6), there exists a Gaussian polynomial $f \in R[t]$ such that $c(f)$ is a nonprincipal ideal of $R$. However, a polynomial extension in two variables over $R$ is approximately Gorenstein [H, (1.14)]. Therefore, by Theorem 1.5, $f$ regarded as a polynomial over this larger ring is not Gaussian.

\section{Gaussian for polynomials of Degree at most $n$}

In this section we prove a sharpening of Theorem (1.5). The socle of $R$ is the ideal spanned by the simple submodules of $R$. If $R$ is local with maximal ideal $\mathbf{m}$, then $(0: \mathbf{m})$ is the socle of $R$. In the case where $(R, \mathbf{m})$ is a zero-dimensional local Gorenstein ring, the socle of $R$ is principal and $R$ is an essential extension of its socle.

Theorem 2.1. Let $(R, \mathbf{m})$ be a zero-dimensional local Gorenstein ring and let $y R$ denote the socle of $R$. Suppose $f(t) \in R[t]$ is such that $\operatorname{deg}(f)=n$ and $c(f)$ is not principal. Then there exists a polynomial $g(t) \in R[t]$ such that $\operatorname{deg}(g) \leq n, f g=0$, and $c(f) c(g)=y R$. In particular, $f$ is not Gaussian for polynomials of degree at most n.

Proof. Let $f(t)=a_{n} t^{n}+a_{n-1} t^{n-1}+\ldots+a_{1} t+a_{0} \in R[t]$. The condition for another polynomial $g(t)=b_{m} t^{m}+\ldots+b_{1} t+b_{0}$ that the product $f g=0$ is clearly equivalent 
to a system of $n+m+1$ linear equations in the $m+1$ "variables" $b_{m}, \ldots, b_{1}, b_{0}$,

$$
\begin{aligned}
& a_{n} b_{m}=0, \\
& a_{n-1} b_{m}+a_{n} b_{m-1}=0, \\
& a_{n-2} b_{m}+a_{n-1} b_{m-1}+a_{n} b_{m-2}=0, \\
& \vdots \\
& a_{0} b_{0}=0
\end{aligned}
$$

and in general for each $d$ with $0 \leq d \leq m+n$,

$$
\sum_{i+j=d} a_{i} b_{j}=0 .
$$

With $m=n$ the matrix of coefficients $A$ is $2 n+1 \times n+1$ and may be displayed as

$$
A=\left(\begin{array}{ccccc}
a_{n} & 0 & 0 & \ldots & 0 \\
a_{n-1} & a_{n} & 0 & \ldots & 0 \\
a_{n-2} & a_{n-1} & a_{n} & \ldots & 0 \\
\vdots & \vdots & \vdots & \ddots & \vdots \\
a_{0} & a_{1} & a_{2} & \ldots & a_{n} \\
0 & a_{0} & a_{1} & \ldots & a_{n-1} \\
\vdots & \vdots & \vdots & \ddots & \vdots \\
0 & 0 & 0 & \ldots & a_{0}
\end{array}\right)
$$

Since $c(f)$ is not principal, the dimension of $c(f) / \mathbf{m} c(f)$ over the residue field $R / \mathbf{m}$ is a positive integer $s \geq 2$. By duality [BH, Proposition 3.2.12], it follows that $((0): c(f))=I$ is such that $(I: \mathbf{m}) / I$ has dimension $s$ over $R / \mathbf{m}$. Let $x_{1}, \ldots x_{s}$ be the preimages in $(I: \mathbf{m})$ of a basis for $(I: \mathbf{m}) / I$. Let $c_{i j} \in R$ be such that

$$
a_{i} x_{j}=c_{i j} y \quad \text { for } \quad 0 \leq i \leq n \quad \text { and } \quad 1 \leq j \leq s .
$$

For indeterminates $\left\{z_{i j}\right\}_{0 \leq i \leq n, 1 \leq j \leq s}$ over $R$, let $h(t)=w_{n} t^{n}+\cdots+w_{1} t+w_{0}$, where

$$
w_{i}=z_{i 1} x_{1}+\cdots+z_{i s} x_{s} \quad \text { for } \quad 0 \leq i \leq n .
$$

The product $f h=0$ is equivalent to the system of linear equations: $A \vec{w}=\overrightarrow{0}$, where $\vec{w}$ is the transpose of $\left(w_{n}, \ldots, w_{0}\right)$. Then

$$
\begin{aligned}
a_{n} w_{n}=0 & \Longrightarrow \text { by }(2.3) \quad a_{n}\left(z_{n 1} x_{1}+\ldots+z_{n s} x_{s}\right)=0 \\
& \Longrightarrow \text { by }(2.2) \quad\left(c_{n 1} z_{n 1}+\ldots+c_{n s} z_{n s}\right) y=0,
\end{aligned}
$$

and

$$
\begin{aligned}
a_{n-1} w_{n}+a_{n} w_{n-1}=0 \Longrightarrow & a_{n-1}\left(z_{n 1} x_{1}+\ldots+z_{n s} x_{s}\right) \\
& +a_{n}\left(z_{n-11} x_{1}+\ldots+z_{n-1 s} x_{s}\right)=0,
\end{aligned}
$$

which by $(2.2)$ implies $\left(\sum_{i=1}^{s}\left(c_{n-1 i} z_{n i}+c_{n i} z_{n-1 i}\right)\right) y=0$. And in general for each $d$ with $0 \leq d \leq 2 n$,

$$
\sum_{i+j=d} a_{i} w_{j}=0 \Longrightarrow \text { by }(2.3) \quad \sum_{i+j=d} a_{i}\left(z_{j 1} x_{1}+\ldots+z_{j s} x_{s}\right)=0
$$


which by (2.2) implies

$$
\left(\sum_{i+j=d}\left(c_{i 1} z_{j 1}+\ldots+c_{i s} z_{j s}\right)\right) y=0, \quad 0 \leq d \leq 2 n .
$$

Since $y$ is a socle element of $R$, to solve the system of equations (2.4) over $R$ in the variables $\left\{z_{i j}\right\}$ is equivalent to solving over the residue field of $R$ the system

$$
\sum_{i+j=d}\left(c_{i 1}^{\prime} z_{j 1}+\ldots+c_{i s}^{\prime} z_{j s}\right)=0, \quad 0 \leq d \leq 2 n,
$$

of $2 n+1$ homogeneous linear equations in the $s(n+1) \geq 2 n+2$ variables $\left\{z_{i j}\right\}$, where $c_{i j}^{\prime}$ denotes the image in $R / \mathbf{m}$ of $c_{i j} \in R$. Let $z_{i j}=e_{i j}^{\prime}$ be a nontrivial solution of (2.5) over $R / \mathbf{m}$, and let $e_{i j} \in R$ be a preimage of $e_{i j}^{\prime}$ for $0 \leq i \leq n, 1 \leq j \leq s$. Then for $g(x)=b_{n} t^{n}+\ldots+b_{1} t+b_{0}$, where $b_{i}=e_{i 1} x_{1}+\ldots+e_{i s} x_{s}$, we have $f g=0$ in $R[t]$. The linear independence of the images of the $x_{i}$ in $(I: \mathbf{m}) / I$ implies that $c(g)$ is not contained in $I$. Hence $c(f) c(g)=y R \neq 0$.

Corollary 2.6. Let $(R, \mathbf{m})$ be a local Noetherian approximately Gorenstein ring. If $f(t) \in R[t]$ with $\operatorname{deg}(f)=n$ is such that $c(f)$ is minimally generated by $s \geq 2$ elements, then $f$ fails to be Gaussian with respect to polynomials of degree $\left\lfloor\frac{n}{s-1}\right\rfloor$.

Proof. As in the proof of $(1.5)$, one may reduce to the case where $(R, \mathbf{m})$ is zerodimensional local and Gorenstein. It suffices to prove there is a polynomial $g(t) \in$ $R[t]$ such that $\operatorname{deg}(g)=m=\left\lfloor\frac{n}{s-1}\right\rfloor$ and $0=c(f g) \neq c(f) c(g)$. This is equivalent to showing the existence of a nontrivial solution to a system of $n+m+1$ homogeneous linear equations over $R / \mathbf{m}$ in $s(m+1)$ variables. If $s(m+1)>n+m+1$ such a nontrivial solution always exists. However, $s(m+1) \leq n+m+1$ iff $m+1 \leq \frac{n}{s-1}$, which is false for $m=\left\lfloor\frac{n}{s-1}\right\rfloor$.

Corollary 2.7. Let $R$ be a ring which is locally Noetherian and locally approximately Gorenstein and let $f \in R[t]$ be a polynomial with $\operatorname{deg}(f)=n \geq 0$. If $f$ is Gaussian for polynomials of degree at most $n$, then $f$ has locally principal content ideal and therefore is Gaussian. If $f$ is a regular element, then the content of $f$ is an invertible ideal of $R$.

Proof. As in the proof of (1.5), one reduces to the local case and then to the case where $R$ is a zero-dimensional local Gorenstein ring. Theorem 2.1 then implies that $c(f)$ is principal. By (1.1), it follows that $f$ is Gaussian, and if $f$ is regular, then $c(f)$ is invertible.

Remark 2.8. The proof of Theorem 2.1 can be extended to show the following. Suppose $(R, \mathbf{m})$ is a local Artinian ring. Let $\mathbf{s}=(0: \mathbf{m})$ denote the socle of $R$, and let $s$ be the dimension of $\mathbf{s}$ as a vector space over $R / \mathbf{m}$. Suppose $f(t) \in R[t]$ is a polynomial of degree $n$ and let $I=\left((0):_{R} c(f)\right)$. If the socle dimension, $\operatorname{dim}_{R / \mathbf{m}}((I: \mathbf{m}) / I)$, of $R / I$ is an integer $r>s$, then $f$ is not Gaussian for polynomials of degree $m$, where $m$ is such that $(m+1) r>(n+m+1) s$.

\section{Gaussian polynomials OVER LOCALly NoEtherian RingS}

To extend our results to all locally Noetherian rings we use the following.

Proposition 3.1. Let $(R, \mathbf{m})$ be a local Noetherian ring and let $(\widehat{R}, \widehat{\mathbf{m}})$ denote the $\mathbf{m}$-adic completion of $R$. A polynomial $f(t) \in R[t]$ is Gaussian over $R$ (resp. 
Gaussian for polynomials of degree at most $n$ over $R$ ) if and only if $f(t)$ is Gaussian over $\widehat{R}$ (resp. Gaussian for polynomials of degree at most $n$ over $\widehat{R}$ ).

Proof. Since all ideals of $R$ are closed in the $\mathbf{m}$-adic topology on $R$, a polynomial $f \in R[t]$ is Gaussian over $R$ (resp. Gaussian for polynomials of degree at most $n$ over $R$ ) if and only if for each positive integer $k$ the image of $f$ in $\left(R / \mathbf{m}^{k}\right)[t]$ is Gaussian over $R / \mathbf{m}^{k}$ (resp. Gaussian for polynomials of degree at most $n$ over $\left.R / \mathbf{m}^{k}\right)$. This follows from (1.2) and the fact that if there exists $g \in R[t]$ such that $c(f g) \subsetneq c(f) c(g)$, then $c(f g)+\mathbf{m}^{k} \subsetneq c(f) c(g)+\mathbf{m}^{k}$ for some positive integer $k$. Hence the image of $f$ in $\left(R / \mathbf{m}^{k}\right)[t]$ fails to be Gaussian. Moreover, if $\operatorname{deg}(g) \leq n$, then the image of $f$ fails to be Gaussian for polynomials of degree at most $n$. Since $R / \mathbf{m}^{k} \cong \widehat{R} / \widehat{\mathbf{m}}^{k}$ for each $k$, a polynomial $f \in R[t]$ is Gaussian over $R$ (resp. Gaussian for polynomials of degree at most $n$ over $R$ ) if and only if $f$ is Gaussian over $\widehat{R}$ (resp. Gaussian for polynomials of degree at most $n$ over $\widehat{R}$ ).

Using the fact from (1.3) that a complete local Noetherian reduced ring of positive dimension is approximately Gorenstein, we prove the following:

Theorem 3.2. Let $(R, \mathbf{m})$ be a complete local Noetherian ring and let

$$
f(t)=a_{n} t^{n}+a_{n-1} t^{n-1}+\cdots+a_{1} t+a_{0}
$$

be a polynomial of degree $n$. Assume that $f(t)$ is not a zero divisor and is Gaussian for polynomials of $R[t]$ of degree at most $n$. Then the content ideal $c(f)=$ $\left(a_{n}, \ldots, a_{1}, a_{0}\right) R$ of $f$ is principal.

Proof. Since $f(t)$ is not a zero divisor, at least one of the coefficients of $f$ is a regular element of $R$. If $c(f)=R$, then $c(f)$ is principal, so we assume $c(f) \subseteq \mathbf{m}$. It follows that $\operatorname{dim}(R) \geq 1$. Let $\mathbf{n}$ denote the nilradical of $R$. By Corollary 2.7, the image of $f$ in $(R / \mathbf{n})[t]$ has principal content ideal. Choose an element $a \in R$ which generates $c(f)$ modulo the nilradical $\mathbf{n}$. Since $R$ is local we may assume that $a=a_{k}$, one of the coefficients of $f$. The element $a$ cannot be a zero-divisor else the content of $f$ would be contained in an associated prime of $R$. Let $T$ denote the total quotient ring of $R$, let $j$ be a positive integer and let $I_{j}=\mathbf{n}^{j} T \cap R$. We note that $I_{j}^{2} \subseteq I_{j+1}$, and $a$ is not a zerodivisor on $R / I_{j}$ for all $j$.

By induction on $j$ we prove that $c(f) \subseteq a R+I_{j}$. Since $I_{j}=0$ for large $j$, this will finish the proof. To begin the induction observe that $c(f) \subseteq a R+I_{1}=a R+\mathbf{n}$, by choice of $a$. Assume that $c(f) \subseteq a R+I_{j}$. For each $i \in\{0, \ldots, n\}$, we can write $a_{i}=r_{i} a+c_{i}$, where $r_{i} \in R$ and $c_{i} \in I_{j}$. For $i=k$, we do this with $r_{k}=1$ and $c_{k}=0$. Let

$$
f_{1}(t)=\sum_{i=0}^{n} r_{i} t^{i} \quad \text { and } \quad f_{2}(t)=\sum_{i=0}^{n} c_{i} t^{i} .
$$

Then $f(t)=a f_{1}(t)+f_{2}(t)$. Since $c_{k}=0$, we have $c(f)=a R+c\left(f_{2}\right)$. Let $g(t):=a f_{1}(t)-f_{2}(t)$. Then $c(g)=a R+c\left(f_{2}\right)$, and $f g=a^{2} f_{1}^{2}+f_{2}^{2}$. Therefore $c(f g) \subseteq a^{2} R+I_{j+1}$. Since $f$ is Gaussian for polynomials of degree at most $n$, we have $a^{2} R+a c\left(f_{2}\right)+c\left(f_{2}\right)^{2}=c(f) c(g)=c(f g) \subseteq a^{2} R+I_{j+1}$. Hence $a c\left(f_{2}\right) \subseteq a^{2} R+I_{j+1}$. Since $a$ is a regular element modulo $I_{j+1}$, it follows that $c\left(f_{2}\right) \subseteq a R+I_{j+1}$. Then $c(f) \subseteq a R+I_{j+1}$, which finishes the induction and the proof.

Theorem 3.3. Let $R$ be a locally Noetherian ring and let $f(t) \in R[t]$ be a regular polynomial of degree $n$. If $f$ is Gaussian for polynomials of degree at most $n$, then $f$ is Gaussian and has invertible content ideal. 
Proof. By (1.1), we may reduce to the local case and assume that $(R, \mathbf{m})$ is a local Noetherian ring. By (3.1), we may pass to the $\mathbf{m}$-adic completion $\widehat{R}$ of $R$. Theorem 3.2 then implies that $c(f)$ is principal. Therefore $f$ is Gaussian and has invertible content ideal.

The following corollary is an immediate consequence of Theorem 3.3.

Corollary 3.4. Let $R$ be a locally Noetherian integral domain. A polynomial $f(t) \in$ $R[t]$ of degree $n \geq 0$ has an invertible content ideal if $c(f g)=c(f) c(g)$ for all polynomials $g(t) \in R[t]$ of degree at most $n$.

\section{REFERENCES}

[AK] D. D. Anderson and B. G. Kang, Content formulas for polynomials and power series and complete integral closure, J. Algebra 181 (1996), 82-94. CMP 96:10

[BH] W. Bruns and J. Herzog, Cohen-Macaulay rings, Cambridge University Press, 1993. MR 95h: 13020

[GV] S. Glaz and W. Vasconcelos, The content of Gaussian polynomials, J. Algebra, to appear.

$[\mathrm{H}]$ M. Hochster, Cyclic purity versus purity in excellent Noetherian rings, Trans. Amer. Math. Soc. 231 (1977), 463-488. MR 57:3111

[M] H. Matsumura, Commutative ring theory, Cambridge University Press, 1986. MR 88h:13001

Department of Mathematics, Purdue University, West Lafayette, Indiana 47907-1395

E-mail address: heinzer@math.purdue.edu

E-mail address: huneke@math.purdue.edu 